\title{
Pre-pregnancy BMI, gestational weight gain and postpartum weight retention: a meta-analysis of observational studies
}

\author{
Ke Rong ${ }^{1}$, Kai $\mathrm{Yu}^{2}$, Xiaolong Han ${ }^{3}$, lgnatius MY Szeto ${ }^{2}$, Xueying Qin ${ }^{4}$, Junkuan Wang ${ }^{2}$, \\ Yibing Ning ${ }^{2}$, Peiyu Wang ${ }^{4}$ and Defu $\mathrm{Ma}^{4, *}$ \\ ${ }^{1}$ Department of Orthopedics, Minhang Hospital, Fudan University, Shanghai, People's Republic of China: ${ }^{2}$ Nestlé \\ Research Center, Beijing, People's Republic of China: ${ }^{3}$ China Astronaut Research and Training Center, Department \\ of Space Food and Nutrition, Beijing, People's Republic of China: ${ }^{4}$ School of Public Health, Peking University Health \\ Science Center, 38 Xueyuan Road, Haidian District, Beijing 100191, People's Republic of China
}

Submitted 3 April 2014: Final revision received 4 September 2014: Accepted 8 October 2014: First published online 20 November 2014

\begin{abstract}
Objective: To determine the association of gestational weight gain (GWG) or pre-pregnancy BMI with postpartum weight retention (PPWR).

Design: Meta-analysis.

Setting: PubMed, Cochrane Controlled Trials Register, EMBASE, Science Citation Index Expanded, Current Contents Connects and Biosis Previews were used to search articles.

Subjects: Publications that described the influence of pre-pregnancy BMI or GWG on PPWR.

Results: Seventeen studies that satisfied the eligibility criteria were included in the analyses. Women with inadequate and excessive GWG had significantly lower mean PPWR of $-2 \cdot 14 \mathrm{~kg}(95 \% \mathrm{CI}-2 \cdot 43,-1.85 \mathrm{~kg})$ and higher PPWR of $3.21 \mathrm{~kg}$ (95\% CI $2.79,3.62 \mathrm{~kg}$ ), respectively, than women with adequate GWG. When postpartum time spans were stratified into 1-3 months, 3-6 months, 6-12 months, 12-36 months and $\geq 15$ years, the association between inadequate GWG and PPWR faded over time and became insignificant $(-1.42 \mathrm{~kg} ; 95 \% \mathrm{CI}-3 \cdot 08,0 \cdot 24 \mathrm{~kg})$ after $\geq 15$ years. However, PPWR in women with excess GWG exhibited a U-shaped trend; that is, a decline during the early postpartum time span (year 1) and then an increase in the following period. Meta-analysis of qualitative studies showed a significant relationship between excessive GWG and higher PPWR risk $(\mathrm{OR}=2 \cdot 08 ; 95 \% \mathrm{CI} 1 \cdot 60,2 \cdot 70)$. Moreover, meta-analysis of pre-pregnancy BMI on PPWR indicated that mean PPWR decreased with increasing BMI group.

Conclusions: These findings suggest that GWG, rather than pre-pregnancy BMI, determines the shorter- or longer-term PPWR.
\end{abstract}

Overweight and obesity are associated with increased risks of morbidity and mortality related to CVD, diabetes, kidney diseases and certain cancers ${ }^{(1)}$. In recent decades, the prevalence of both overweight and obesity has been increasing steadily in all age groups worldwide ${ }^{(2)}$. One of the natural and biological causes of weight recycling in women is pregnancy. Pregnancy is a period in most women's lives when substantial weight is gained, considerably altering their future weight-gain trajectory ${ }^{(3)}$. The obesity epidemic has demonstrated that weight gain from pregnancy may lead to obesity development ${ }^{(4)}$. Approximately $10 \%$ to $15 \%$ of women retain the weight they gain during pregnancy on a long-term basis and a number of these ultimately become obese ${ }^{(4)}$. Thus, the pregnancy-postpartum period is critical because it can significantly affect long-term weight management and predispose women to chronic diseases later in life.

Pre-pregnancy BMI, gestational weight gain (GWG) and postpartum weight retention (PPWR) are not only nutritional problems but may also be related to activity, genetic and psychological factors faced by women of childbearing age. According to a review by Gunderson and Abrams, PPWR is presumably due to a combination of several factors, such as dietary intake, lack of physical activity, lactation, smoking status, pre-pregnancy BMI, GWG and parity, and is associated with increased risks of long-term 
obesity for women ${ }^{(5)}$. Among these factors, pre-pregnancy BMI and GWG are the major ones ${ }^{(6)}$. Since the US Institute of Medicine (IOM) published its guidelines for a healthy GWG in 1990, which were updated in 2009, GWG categorization into 'inadequate', 'adequate' and 'excess' has been a commonly used method for predicting PPWR and obesity over short and long terms ${ }^{(7-15)}$. Numerous studies have supported the suitability of GWG guidelines for positive pregnancy outcomes ${ }^{(7-15)}$. However, the postpartum duration in these studies ranged from 0.5 month to 21 years, and the associations between GWG categories and PPWR were found to vary significantly ${ }^{(7-15)}$. Therefore, it is necessary to clarify whether the extent of the association between GWG categories and PPWR is different over the longer term compared with shorter periods of time. In addition, a number of studies have reported a direct association between pre-pregnancy BMI and PPWR, whereas others studies have found no such association $^{(16-19)}$.

The associations among pre-pregnancy BMI, GWG and PPWR should be determined because the validation of such associations can potentially guide targeted intervention efforts for preventing obesity in women. In the present study, we performed a systematic literature research and meta-analysis to determine the effects of GWG in accordance with the IOM guidelines and of pre-pregnancy BMI in accordance with the WHO classification on PPWR. Moreover, we examined whether weight increase during pregnancy, rather than the pre-pregnancy BMI, determines the shorter- or longer-term PPWR.

\section{Experimental methods}

\section{Literature search}

We followed the Meta-analysis of Observational Studies in Epidemiology (MOOSE) guidelines for the conduct of systematic reviews and meta-analyses of observational studies $^{(20)}$. Various databases, namely PubMed (updated up to September 2013), Cochrane Controlled Trials Register, EMBASE (1985 to September 2013), Science Citation Index Expanded, Current Contents Connects (1998 to 2013) and Biosis Previews (1926 to 2013), were used to search articles (in English) that described observational studies of the influence of pre-pregnancy BMI or GWG on PPWR. Titles, abstracts and subject headings in the databases were searched using the following Boolean phrases: ('Pregnant' OR 'Pregnancy' OR 'Prenatal' OR 'Gestation' OR 'Gravidity') AND ('Postpartum' OR 'Post-partum' OR 'Post partum' OR 'Post pregnancy' OR 'Post-natal' OR 'After delivery' OR 'After birth' OR 'After childbirth') AND ('Weight gain' OR 'Weight increase' OR 'Weight gains' OR 'Weight gained' OR 'Gained weight' OR 'Weight growth' OR 'GWG' OR 'BMI') AND ('Retention' OR 'Retain weight' OR 'Maintain weight' OR 'Keep weight' OR 'Stabilization' OR 'Sustain weight' OR 'Upload weight' OR 'PPWR'). In addition, a manual search of reference lists of relevant and related articles was conducted to ensure a complete collection. Moreover, authors of articles were contacted via email when the required data were only partially reported in the published articles to ensure that all necessary data were included.

\section{Study selection}

Studies were selected for analysis if they met all of the following a priori defined inclusion criteria: (i) published in English; (ii) focused on healthy women; (iii) singleton pregnancies; (iv) delivery at term is reported; (v) GWG is classified as above, within or below the IOM recommendation or with very similar cut-off values, and pre-pregnancy BMI is classified as underweight $\left(<18.5 \mathrm{~kg} / \mathrm{m}^{2}\right)$, normal weight $\left(18.5-24.9 \mathrm{~kg} / \mathrm{m}^{2}\right)$, overweight $\left(25.0-29.9 \mathrm{~kg} / \mathrm{m}^{2}\right)$ or obese $\left(\geq 30 \cdot 0 \mathrm{~kg} / \mathrm{m}^{2}\right)$, in accordance with WHO classifications; (vi) PPWR is documented as a continuous variable or an odds ratio between GWG above and within the recommendations; and (vii) PPWR is documented at 4 weeks postpartum and/or later. If the study sample overlapped with that in another article, or if two articles described aspects of the same study, only the publication with the largest sample was used. If a study reported different postpartum time points, these were all included in the meta-analysis.

\section{Data extraction and quality assessment}

The data from original articles were independently abstracted by two reviewers who used a standardized data collection form. Any disagreement between the two reviewers was resolved through discussion with the third reviewer. The following data were collected: (i) first author and publication year; (ii) population information, including country, sample size, gestational age, healthy status and pre-pregnancy BMI; (iii) study characteristics, including study design, definition of GWG and PPWR, and postpartum time; and (iv) information about the outcome, such as PPWR or odds ratio.

Methodological quality of studies was evaluated using the Newcastle-Ottawa Scale for assessing cohort studies used in meta-analyses ${ }^{(21)}$. A star was assigned where follow-up was a minimum of 1 year and where the loss to follow-up had been estimated for the study and reported in the article and where loss was less than $25 \%$. In addition, we conducted meta-regression analyses to assess specific aspects of quality such as time postpartum.

\section{Statistical analysis}

The differences in the mean PPWR of women whose GWG was below or above the recommended values minus the mean PPWR of women who gained weight within the recommended values were used to calculate the mean net change for each subgroup. Each study was weighted by its inverse variance. Weighted mean differences and the corresponding $95 \%$ confidence 
intervals were calculated using a random-effects model for all cases. The heterogeneity of different sizes across trials was tested by means of $Q$ statistics, in which statistical significance was established at $P<0 \cdot 10$. We also calculated the $I^{2}$ statistic, which described the proportion of the total variation that is caused by heterogeneity. Sensitivity analysis was conducted to investigate the influence of a single trial on the overall effect; this effect was estimated by omitting one study in each turn. Moreover, we performed sensitivity analyses excluding studies with self-reported GWG/PPWR or without clear definitions of GWG and PPWR or using the 1990 IOM guidelines. In addition, studies were stratified into five different categories according to the time postpartum (1-3 months, 3-6 months, 6-12 months, $12-36$ months and $\geq 15$ years) or into two different categories according to the different IOM recommendations (IOM 1990 criteria and IOM 2009 criteria) to perform subgroup analysis. Furthermore, we conducted meta-regression analyses to assess whether PPWR differences were related to the time postpartum. Publication bias was assessed with funnel plots, which plotted the standard error of the studies against their corresponding size differences. In addition, Egger's linear regression test and Begg's rank correlation test were conducted to detect publication bias. All the analyses were conducted in the statistical software package STATA version 9.2. $P<0.05$ was considered statistically significant, unless otherwise specified.

\section{Results}

The trial flowchart is illustrated in Fig. 1. Electronic and manual literature searches yielded 1291 results, of which

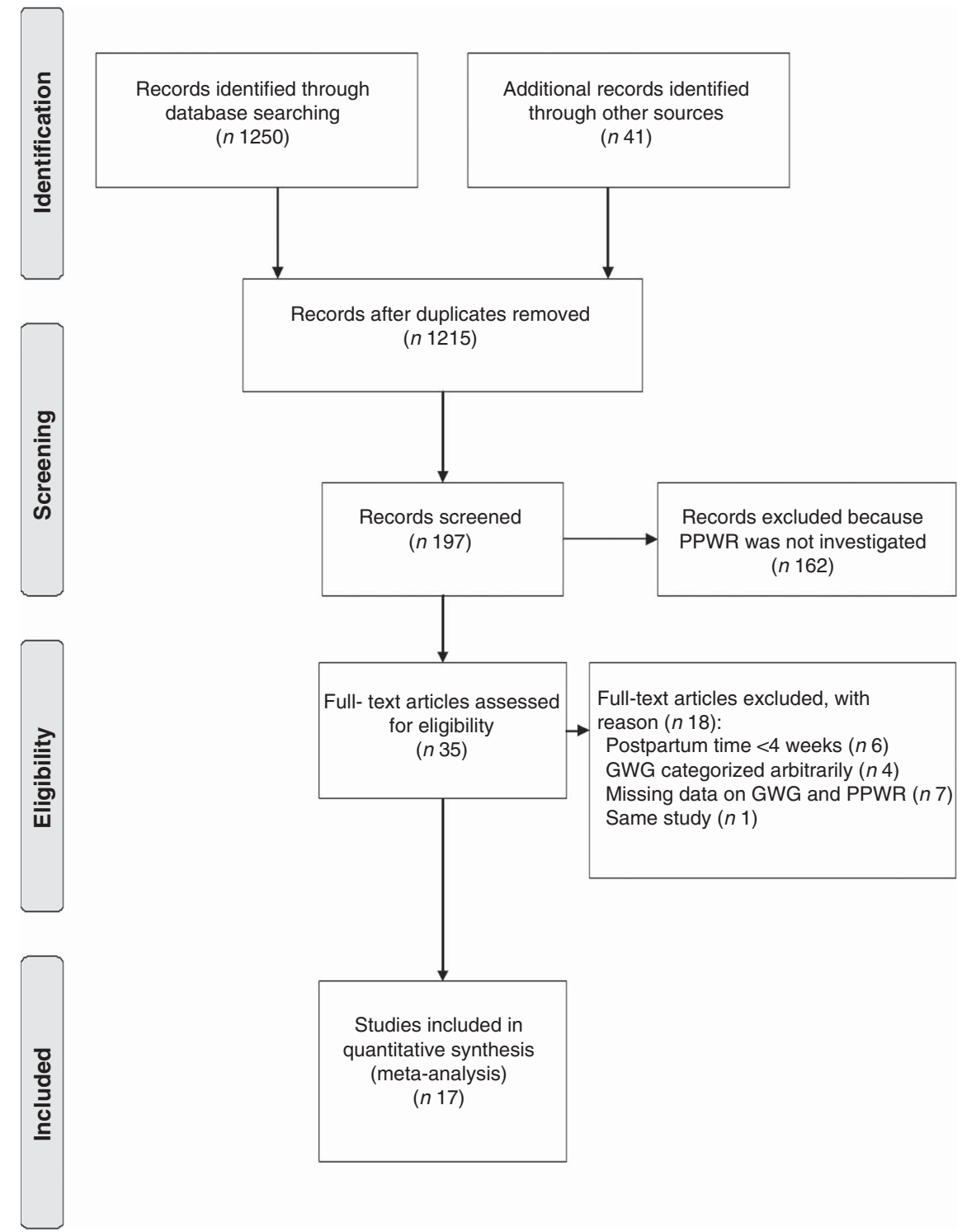

Fig. 1 Flowchart illustrating the selection process for articles included in the present meta-analysis (PPWR, postpartum weight retention; GWG, gestational weight gain) 
Table 1 Main characteristics of the studies included in the present meta-analysis

\begin{tabular}{|c|c|c|c|c|c|c|}
\hline Study ID & Reference & Country & $\begin{array}{l}\text { Time postpartum } \\
\text { (months) }\end{array}$ & Definition of GWG & Definition of PPWR & Indices* \\
\hline Amorim (2007) & Amorim et al. ${ }^{(11)}$ & Sweden & 180 & $\begin{array}{l}\text { Body weight in late pregnancy - pre-pregnancy } \\
\text { body weight }\end{array}$ & $\begin{array}{l}\text { Body weight after pregnancy - pre-pregnancy } \\
\text { body weight }\end{array}$ & 1,2 \\
\hline Althuizen (2011) & Althuizen et al. ${ }^{(25)}$ & Netherlands & 24 & $\begin{array}{l}\text { End-of-pregnancy weight - pre-pregnancy } \\
\text { weight }\end{array}$ & Postpartum weight - pre-pregnancy weight & 3 \\
\hline Begum (2012) & Begum et al. ${ }^{(19)}$ & Canada & 3 & $\begin{array}{l}\text { Highest weight in pregnancy - pre-pregnancy } \\
\text { body weight }\end{array}$ & $\begin{array}{l}\text { Postpartum body weight - pre-pregnancy } \\
\text { body weight }\end{array}$ & 1,2 \\
\hline Baker (2008) & Baker et al. ${ }^{(17)}$ & Denmark & 6,18 & Self-reported & $\begin{array}{l}\text { Current reported weight - pre-pregnancy } \\
\text { weight }\end{array}$ & 2 \\
\hline Huang (2010) & Huang et al. ${ }^{(18)}$ & Taiwan & 6 & Not defined & Not defined & 2 \\
\hline Krause (2010) & Krause et al. ${ }^{(12)}$ & USA & 3,6 & Not defined & $\begin{array}{l}\text { Postpartum weight - pre-pregnancy body } \\
\text { weight }\end{array}$ & 1,2 \\
\hline Kac (2004) & Kac et al. ${ }^{(14)}$ & Brazil & $2,6,9$ & $\begin{array}{l}\text { Self-reported: 'How much weight did you gain } \\
\text { during the last pregnancy?' }\end{array}$ & $\begin{array}{l}\text { Body weight after pregnancy - pre-pregnancy } \\
\text { body weight }\end{array}$ & 1 \\
\hline Koh (2013) & Koh et al. ${ }^{(24)}$ & Singapore & $6,12,24$ & Final pregnancy weight - pre-pregnancy weight & Maternal weight - pre-pregnancy weight & 3 \\
\hline Lowell (2010) & Lowell and Miller ${ }^{(7)}$ & Canada & 5-9 & $\begin{array}{l}\text { Self-reported: 'How much weight did you gain } \\
\text { during your pregnancy?' }\end{array}$ & $\begin{array}{l}\text { Self-reported body weight after pregnancy - } \\
\text { pre-pregnancy body weight }\end{array}$ & 1 \\
\hline Mamum (2010) & Mamum et al. ${ }^{(9)}$ & Australia & 252 & $\begin{array}{l}\text { Maximum weight in pregnancy - pre-pregnancy } \\
\text { weight }\end{array}$ & Postpartum weight gain & 1 \\
\hline Maddah (2009) & Maddah and Nikooyeh ${ }^{(15)}$ & Iran & $24,36,48$ & $\begin{array}{l}\text { Body weight at last antenatal visit - body } \\
\text { weight at first antenatal visit }\end{array}$ & $\begin{array}{l}\text { Body weight after pregnancy - pre-pregnancy } \\
\text { body weight }\end{array}$ & $1,2,3$ \\
\hline Østbye (2010) & Østbye et al. ${ }^{(13)}$ & USA & 33.6 & Not defined & Not defined & 1,2 \\
\hline Oken (2009) & Oken et al. ${ }^{(26)}$ & USA & 12 & $\begin{array}{l}\text { Last clinically measured weight recorded prior } \\
\text { to delivery - pre-pregnancy weight }\end{array}$ & $\begin{array}{l}\text { Weight at } 12 \text { months following delivery - } \\
\text { pre-pregnancy weight }\end{array}$ & 3 \\
\hline Rode (2011) & Rode et al. ${ }^{(8)}$ & Denmark & 24 & $\begin{array}{l}\text { Body weight at } 37 \text { weeks' gestation - } \\
\text { pre-pregnancy weight }\end{array}$ & $\begin{array}{l}\text { Body weight after pregnancy - pre-pregnancy } \\
\text { body weight }\end{array}$ & $1,2,3$ \\
\hline Rothberg (2011) & Gould Rothberg et al. ${ }^{(16)}$ & USA & $1 \cdot 5,6,12$ & Not defined & Not defined & 2 \\
\hline Scholl (1995) & Scholl et al. ${ }^{(23)}$ & USA & $1 \cdot 5,6$ & Not defined & $\begin{array}{l}\text { Measured postpartum weight - pregravid } \\
\text { weight }\end{array}$ & 1 \\
\hline Walker (2004) & Walker et al. ${ }^{(10)}$ & USA & 1.5 & $\begin{array}{l}\text { End pregnancy body weight -pre-pregnancy } \\
\text { body weight }\end{array}$ & $\begin{array}{l}\text { Postpartum body weight - pre-pregnancy } \\
\text { body weight }\end{array}$ & 1,2 \\
\hline
\end{tabular}

GWG, gestational weight gain; PPWR, postpartum weight retention.

*1, PPWR of different GWG categories; 2, PPWR of different pre-pregnancy BMI groups; 3, OR of PPWR $\geq 5 \mathrm{~kg}$ between GWG above recommendation and GWG within recommendation. 
seventy-six articles were excluded because of duplication. A total of 1018 articles were excluded after the preliminary screening performed by going through the titles and abstracts. A total of 162 articles were further excluded on the basis of the inclusion criteria. Thus, thirty-five full-text articles were screened for detailed evaluation, among which seventeen studies were excluded because of having too short a follow-up time ( $<4$ weeks; six studies), the arbitrary categorization of GWG (four studies) and missing data on GWG and PPWR (seven studies). Two studies described the aspects of the same study, but only the study reporting the PPWR was used ${ }^{(14,22)}$. Thus, seventeen studies, two of which were obtained from reference lists, were included in the present meta-analysis ${ }^{(7-19,23-26)}$.

The characteristics of the included trials are shown in Table 1. Four studies were conducted in Europe, eight studies were conducted in North America, three studies were conducted in Asia, one study was conducted in Brazil and one study was conducted in Australia. Socioeconomic status among the studies was considered homogeneous, except for one study that explicitly included low-income women. None of these studies was confined to either under- or overweight women. Women had to be older than 18 years in all except four studies. Two studies reported parity $\geq 1$ as an inclusion criterion. Mean prepregnancy BMI was in the range of 21.7 to $26.4 \mathrm{~kg} / \mathrm{m}^{2}$. An explicit definition for both GWG and PPWR was reported by eleven authors. Maternal pre-pregnancy weight was measured only in one study, whereas the other studies used self-reported pre-pregnancy weight and/or GWG, which may be a source of information bias. Postpartum body weight was measured by the study team in most, but not all, of the studies.

The quality of studies included in meta-analyses was assessed by applying the Newcastle-Ottawa scale for cohort studies. Table 2 presents the scores assigned to each included study. All of the studies had a score of 6 or more (stars) and were higher-scoring studies. The mean score was about 7. Because PPWR as outcome of interest was present at the start of all of the included studies, all of the included studies were not assigned a score for outcome. In addition, studies that had inadequate follow-up time and either did not report losses to follow-up or loss was more than $25 \%$ were also not assigned a score.

In the present research, eleven studies, which involved 67853 women, analysed PPWR at different time points from 1 month to 21 years. Six of the studies reported GWG as recommended by the IOM in 1990, whereas four studies used the updated recommendations from 2009. One study applied the Canadian GWG recommendations of 1999, which are similar to the 1990 IOM guidelines. Compared with women who gained weight within the recommended values, those with GWG below the IOM criteria retained approximately $2 \mathrm{~kg}$ less $(-2 \cdot 14 \mathrm{~kg} ; 95 \% \mathrm{CI}$ $-2.43,-1.85 \mathrm{~kg}$ ) weight (Fig. 2). When postpartum time spans were stratified into 1-3 months, 3-6 months,
Table 2 Quality assessment of studies included in the present meta-analysis using the Newcastle-Ottawa Scale

\begin{tabular}{|c|c|c|c|}
\hline Study ID & Selection & Comparability & Outcome/exposure \\
\hline Amorim (2007) & 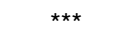 & ** & 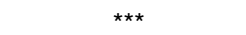 \\
\hline Althuizen (2011) & $\star \star \star *$ & ** & ** \\
\hline Begum (2012) & 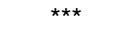 & ** & ** \\
\hline Baker (2008) & 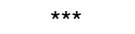 & ** & ** \\
\hline Huang (2010) & 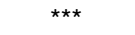 & ** & * \\
\hline Krause (2010) & 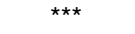 & ** & * \\
\hline $\mathrm{Kac}(2004)$ & 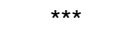 & ** & * \\
\hline Koh (2013) & 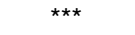 & ** & ** \\
\hline Lowell (2010) & $\star \star \star *$ & ** & * \\
\hline Mamum (2010) & 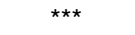 & ** & ** \\
\hline Maddah (2009) & $\star \star \star *$ & ** & ** \\
\hline Østbye (2010) & *** & ** & ** \\
\hline Oken (2009) & 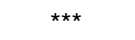 & ** & ** \\
\hline Rode (2011) & 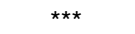 & ** & *** \\
\hline Rothberg (2011) & 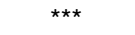 & ** & ** \\
\hline Scholl (1995) & $* \star \star$ & ** & * \\
\hline Walker (2004) & *** & $\star *$ & ** \\
\hline
\end{tabular}

6-12 months, 12-36 months and $\geq 15$ years, the association faded over time and became insignificant $(-1.42 \mathrm{~kg}$; $95 \% \mathrm{CI}-3 \cdot 08,0 \cdot 24 \mathrm{~kg}$ ) after $\geq 15$ years (Table 3 ). We next performed meta-regression analysis to assess whether PPWR differences were related to the time postpartum. Although there was no significant impact, there was a trend towards gradual reduction in PPWR differences with the extension of time $(P=0 \cdot 08)$. Compared with women who had adequate GWG, those with excess GWG retained an additional $3.21 \mathrm{~kg}$ (95\% CI $2.79,3.62 \mathrm{~kg}$ ) weight (Fig. 2). Subgroup analysis with respect to postpartum time spans revealed that, in contrast to PPWR in women with adequate GWG, PPWR in women with excess GWG showed a U-shaped trend; that is, a decline during the early postpartum period (year 1 ; from $4.33 \mathrm{~kg}$ at 3 months to $2.11 \mathrm{~kg}$ at 1 year) and then an increase in the follow-up period (from $2 \cdot 11 \mathrm{~kg}$ at 1 year to $4.65 \mathrm{~kg}$ at $\geq 15$ years). Sensitivity analyses excluding studies with self-reported GWG/PPWR or without clear definitions of GWG and PPWR did not find any significant differences (data not shown). The exclusion of studies that used the 1990 IOM guidelines in sensitivity analysis did not substantially change the findings (Table 3). In addition, sensitivity analyses with each study removed individually suggested that no study individually altered the pooled results of PPWR significantly. Five studies provided the odds ratio of a weight increase in PPWR of at least $5 \mathrm{~kg}$ in women with excessive GWG and in women with adequate GWG. We combined these odds ratio values and the summarized result showed a significant relationship between excessive GWG and higher PPWR risks (OR=2.08; $95 \%$ CI 1.60, 2.70; Fig. 3).

In the present research, ten studies, which involved 116735 women, analysed the PPWR at different prepregnancy BMI from 1 month to 15 years. Mean PPWR decreased with increasing BMI group. Compared with normal-weight women, underweight women retained an additional $0.54 \mathrm{~kg}$ (95\% CI 0.06, $1.02 \mathrm{~kg}$ ) weight; whereas 
(a)

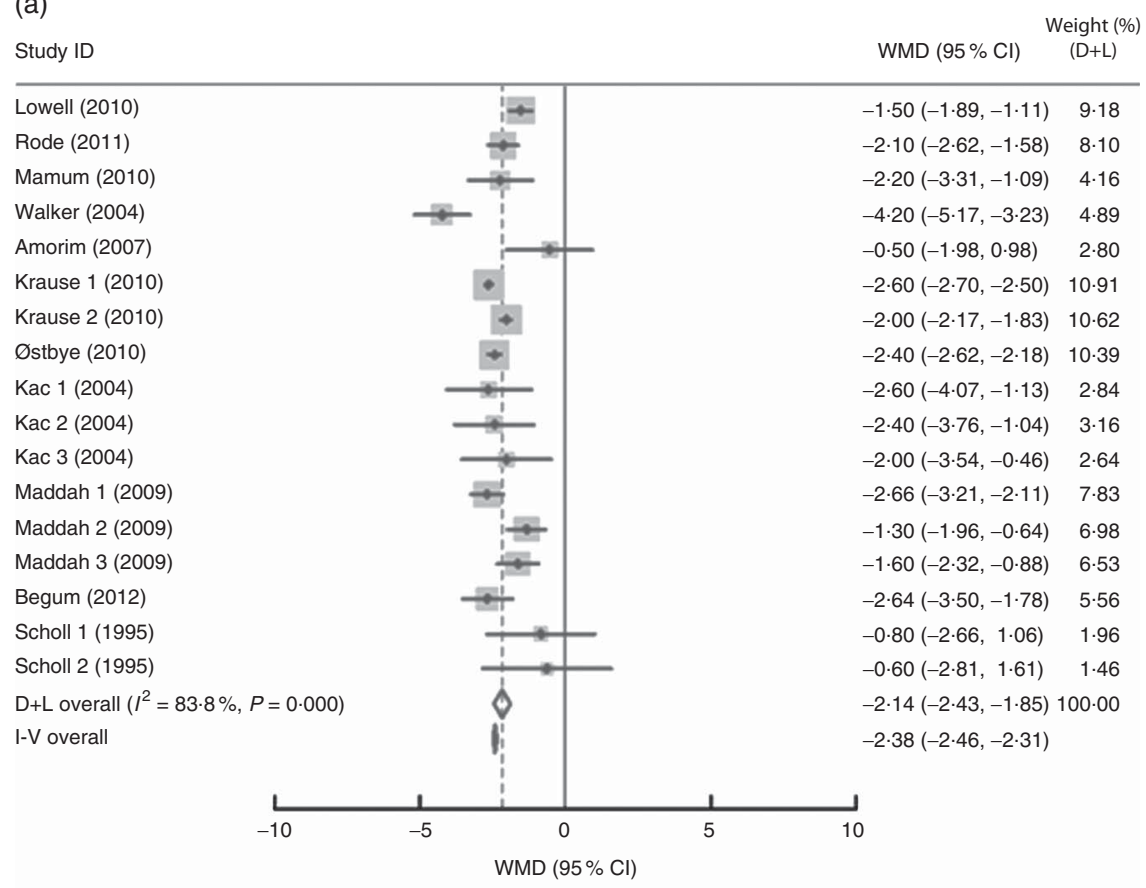

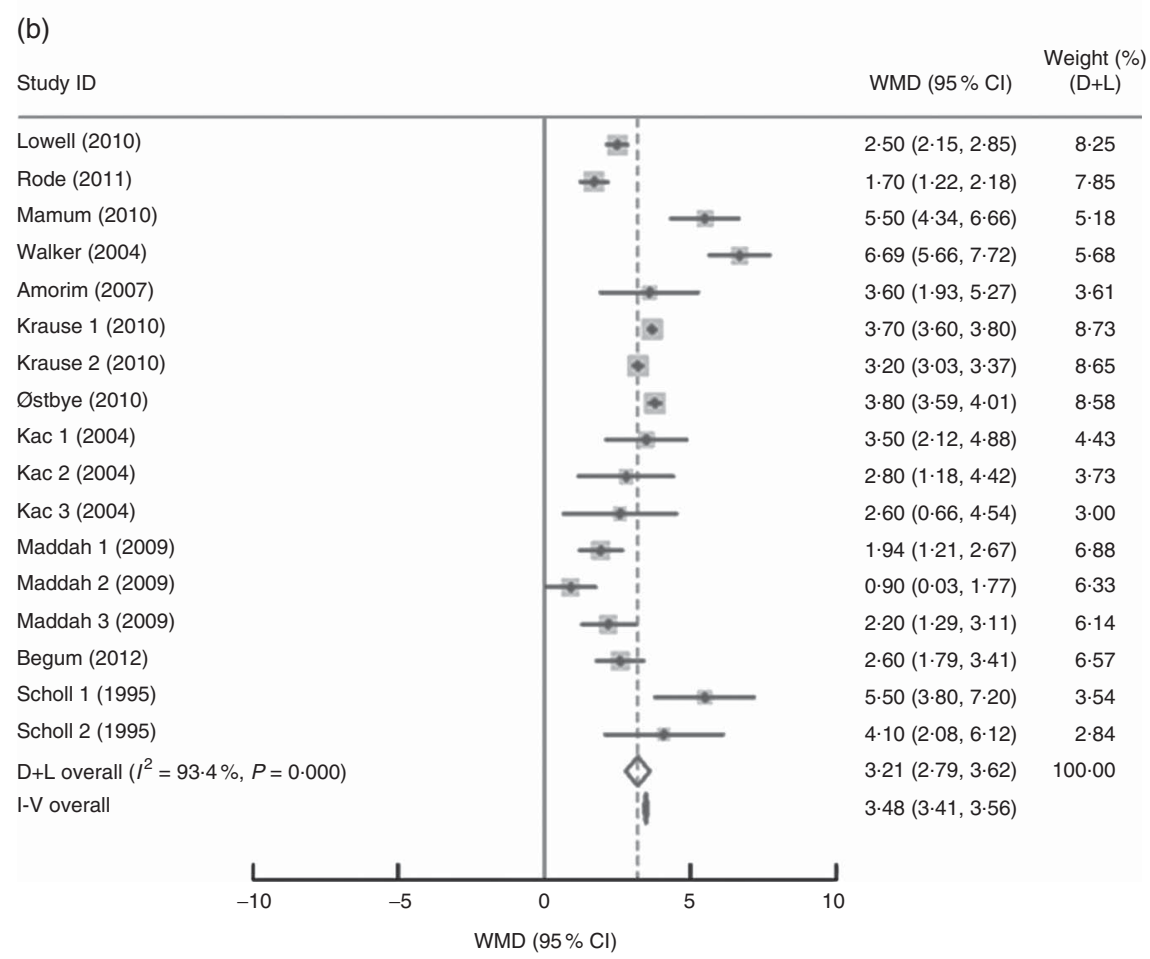

Fig. 2 Pooled estimates for the weighted mean differences (WMD, kg) of postpartum weight retention between women who gained below (a) or above (b) and within the Institute of Medicine recommendation for gestational weight gain. The study-specific WMD and $95 \% \mathrm{Cl}$ are represented by the grey square and horizontal line, respectively; the size of the data marker (grey square) is proportional to the weight of the study in the meta-analysis. The centre of the open diamond presents the pooled WMD and its width represents the pooled $95 \% \mathrm{Cl}$

overweight and obese women had significantly lower PPWR of $-0.81 \mathrm{~kg}(95 \% \mathrm{CI}-1.23,-0.39 \mathrm{~kg})$ and $-2.34 \mathrm{~kg}$ (95\% CI $-3 \cdot 28,-1 \cdot 40 \mathrm{~kg}$ ), respectively (Fig. 4). Subgroup analysis with respect to postpartum time spans did not show any significant differences between the shortand long-term effects of pre-pregnancy BMI on PPWR. 
Table 3 Subgroup analyses of postpartum weight retention stratified by postpartum duration and US Institute of Medicine (IOM) guidelines

\begin{tabular}{|c|c|c|c|c|c|c|c|}
\hline \multirow[b]{2}{*}{ Variable } & \multirow[b]{2}{*}{$n^{*}$} & \multicolumn{3}{|c|}{ Below $v$. within } & \multicolumn{3}{|c|}{ Above $v$. within } \\
\hline & & Net change & $95 \% \mathrm{Cl}$ & $I^{2}(\%)$ & Net change & $95 \% \mathrm{Cl}$ & $I^{2}(\%)$ \\
\hline \multicolumn{8}{|c|}{ Postpartum duration } \\
\hline $1-3$ months & 5 & $-2 \cdot 75$ & $-3.48,-2.03$ & 71.5 & $4 \cdot 33$ & $3.12,5.53$ & $90 \cdot 8$ \\
\hline 3-6 months & 3 & $-2 \cdot 00$ & $-2 \cdot 17,-1 \cdot 83$ & 0.0 & $3 \cdot 20$ & $3.03,3.37$ & 0.0 \\
\hline $6-12$ months & 4 & -1.95 & $-2 \cdot 21,-1 \cdot 68$ & 74.4 & $2 \cdot 11$ & $1 \cdot 61,2 \cdot 61$ & 60.4 \\
\hline $12-36$ months & 3 & -1.82 & $-2.59,-1.05$ & 84.5 & $2 \cdot 33$ & $0.44,4.23$ & $96 \cdot 0$ \\
\hline$\geq 15$ years & 2 & -1.42 & $-3.08,0.24$ & $69 \cdot 1$ & 4.65 & $2 \cdot 8,6.5$ & $70 \cdot 1$ \\
\hline \multicolumn{8}{|l|}{ IOM guidelines } \\
\hline IOM 1990 & 12 & $-2 \cdot 02$ & $-2 \cdot 65,-1 \cdot 38$ & $75 \cdot 3$ & 3.55 & $2 \cdot 34,4 \cdot 76$ & $90 \cdot 6$ \\
\hline IOM 2009 & 5 & $-2 \cdot 33$ & $-2 \cdot 65,-2 \cdot 01$ & 88.9 & 3.08 & $2.61,3.56$ & $95 \cdot 7$ \\
\hline
\end{tabular}

*Number of comparisons.

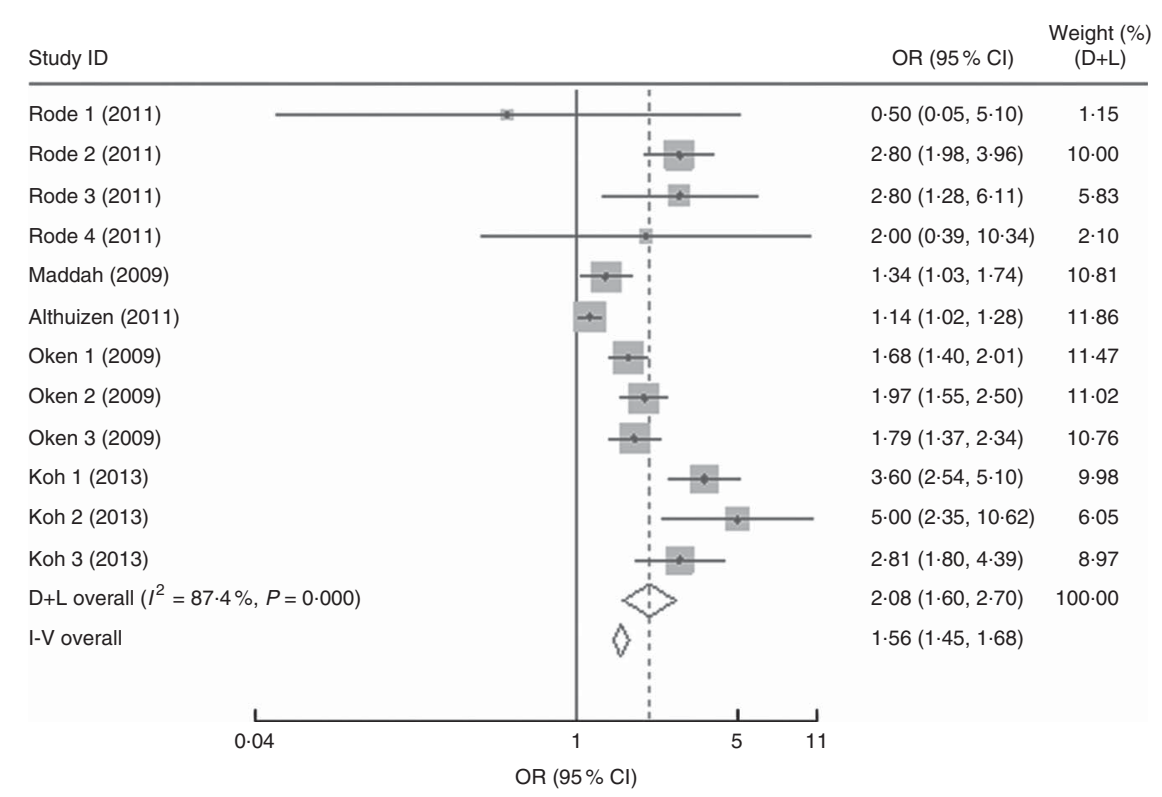

Fig. 3 Forest plot of the studies on the risk of postpartum weight retention of $\geq 5 \mathrm{~kg}$ for the women with excessive gestational weight gain (GWG) $v$. the women with adequate GWG. The study-specific OR and $95 \% \mathrm{Cl}$ are represented by the grey square and horizontal line, respectively; the size of the data marker (grey square) is proportional to the weight of the study in the meta-analysis (note: weights are from random-effects analysis). The centre of the open diamond presents the pooled OR and its width represents the pooled $95 \% \mathrm{Cl}$

Sensitivity analyses with each study removed individually suggested that no study individually altered the pooled results of PPWR significantly.

Potential publication bias was detected using funnel plots, Egger's test and Begg's test. The funnel plots showed a symmetric distribution of PPWR around the summary estimate and thus did not indicate any publication bias (data not shown). Egger's and Begg's tests yielded results that were similar to that of the funnel plots: inadequate $v$. adequate (Egger $P=0.134$; Begg $P=0.869$ ), excessive $v$. adequate (Egger $P=0.312$; Begg $P=0.323$ ), underweight $v$. normal weight (Egger $P=0.901$; Begg $P=0.787$ ), overweight $v$. normal weight (Egger $P=0.858$; Begg $P=0.242$ ) and obese $v$. normal weight (Egger $P=0.739$; Begg $P=0 \cdot 1$ ). In addition, the funnel plot showing the relationship between the odds ratio and the standard error of the logarithmic odds ratio suggested no evidence of publication bias.

\section{Discussion}

The current review presents an update of the metaanalysis by Nehring et al. that compared the effects of GWG in accordance with the IOM guidelines on PPWR ${ }^{(27)}$. Moreover, we determined the effects of pre-pregnancy BMI in accordance with the WHO classification on PPWR and examined whether weight increase during pregnancy, rather than the pre-pregnancy BMI, determines the shorter- or longer-term PPWR for the first time. In the present research, meta-analysis on the influence of GWG on PPWR showed that excessive GWG can significantly 
increase higher PPWR risks ( $\mathrm{OR}=2 \cdot 08,95 \% \mathrm{CI} 1 \cdot 60,2 \cdot 70)$. In addition, the results suggested that mean PPWR decreased with increasing BMI group.

Subgroup analysis of postpartum follow-up time revealed that the association between inadequate GWG and PPWR faded over time and became insignificant $(-1.42 \mathrm{~kg} ; 95 \%$ CI $-3.08,0.24 \mathrm{~kg})$ after $\geq 15$ years. This result supports the findings of the other meta-analysis, which reported that the effect size of this association appeared to decrease over postpartum time, ultimately becoming statistically insignificant after 15 years postpartum $^{(27)}$. In another meta-analysis, Mannan et al. reported that the difference in PPWR in women with inadequate GWG persisted for a long time, and meta-analysis of those studies conducted after 15 years postpartum suggested consistency, albeit slightly decreased effect size $(-1.53 \mathrm{~kg}$; $95 \%$ CI $-2.53,-0.54 \mathrm{~kg}$ ), but remained statistically significant ${ }^{(28)}$. Although the underlying mechanisms of

(a)

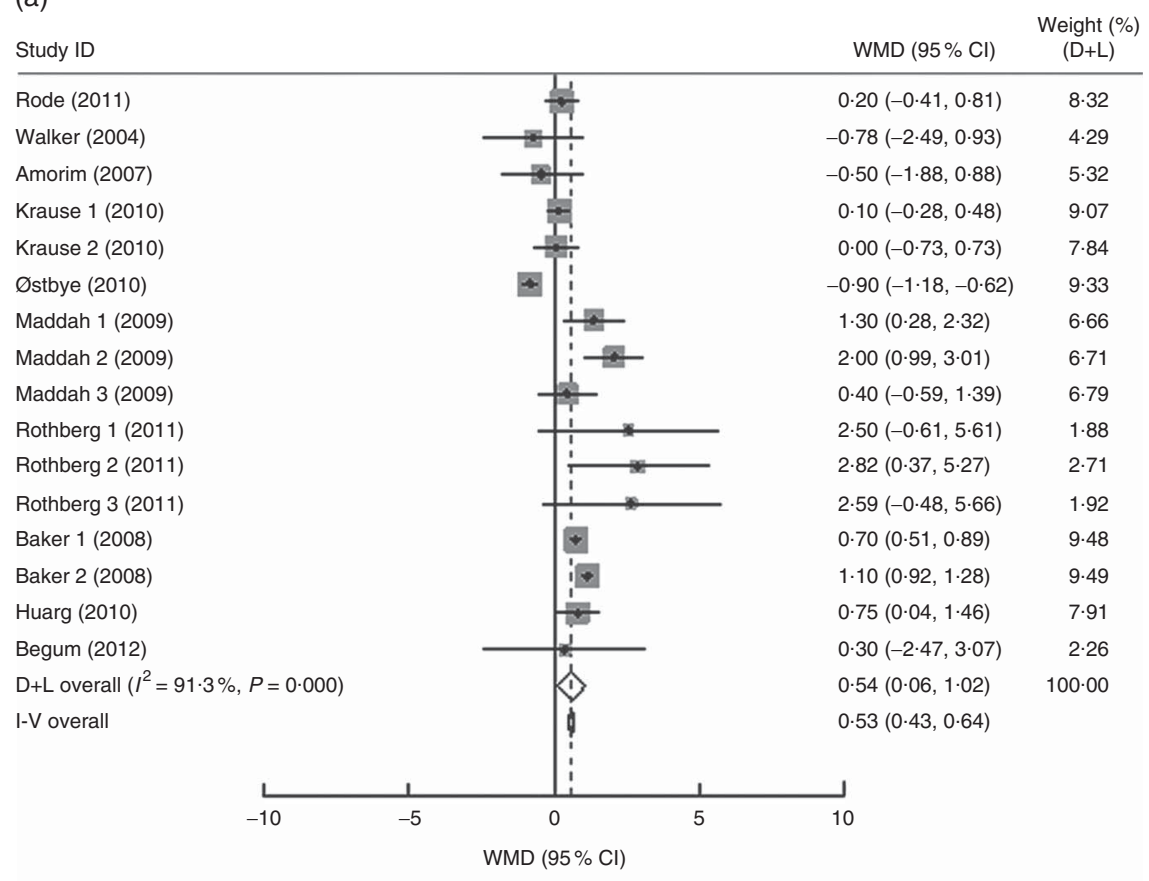

(b)

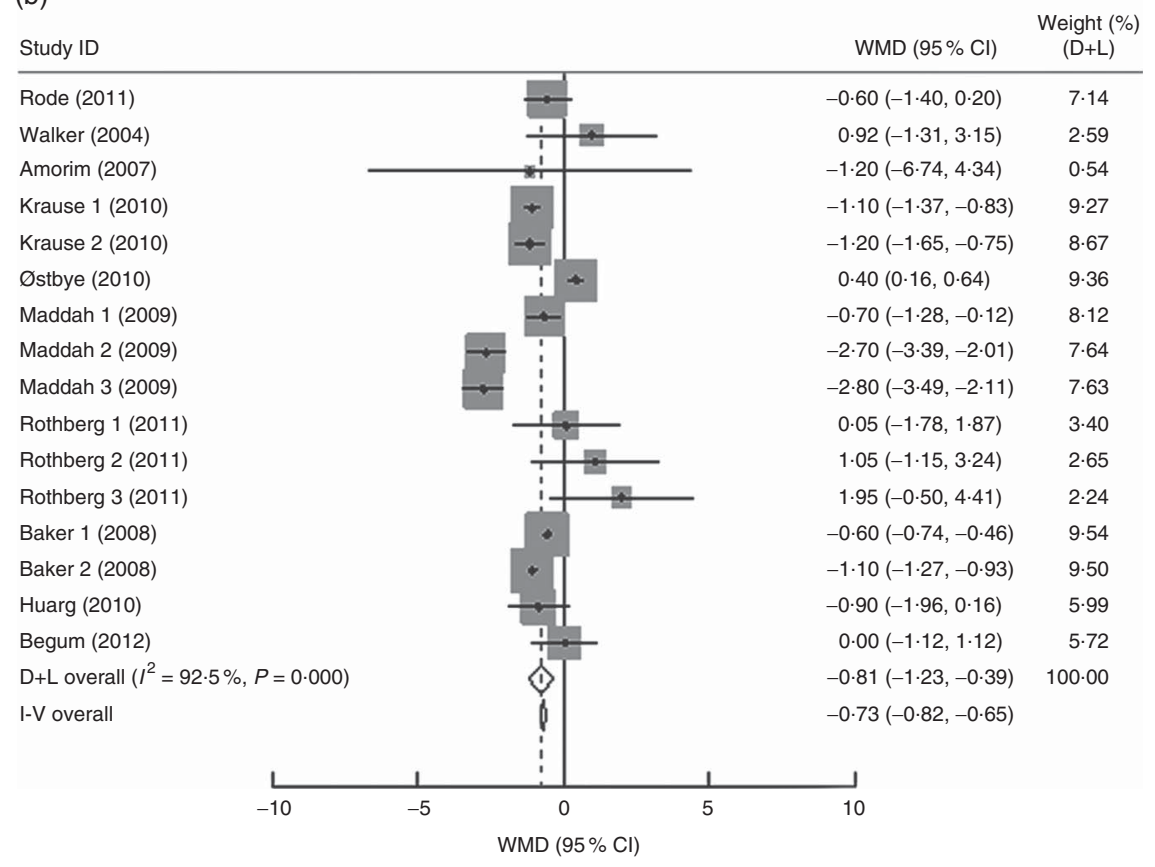

Fig. 4 (Continued on following page) 
(c)

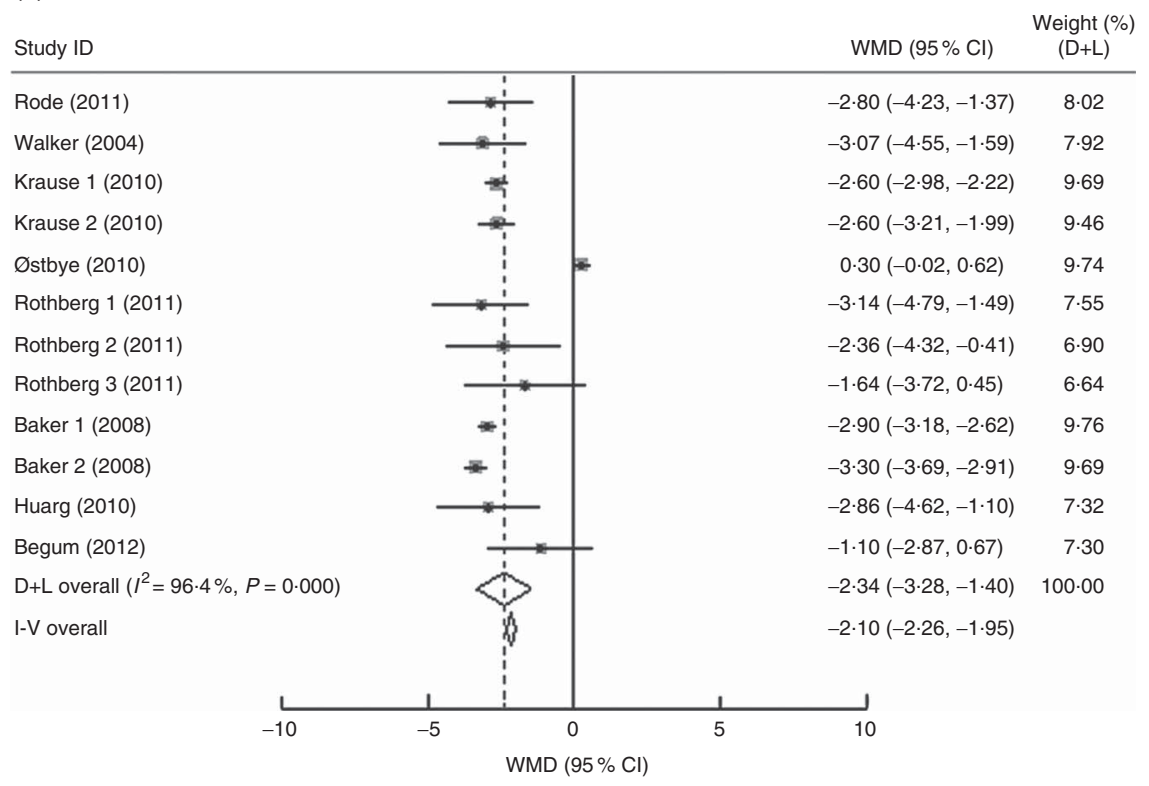

Fig. 4 (Continued from previous page) Pooled estimates for the weighted mean differences (WMD, kg) of postpartum weight retention between women whose pre-pregnancy BMI was less than $18.5 \mathrm{~kg} / \mathrm{m}^{2}$ (a), $25.0-29.9 \mathrm{~kg} / \mathrm{m}^{2}$ (b) or $\geq 30.0 \mathrm{~kg} / \mathrm{m}^{2}$ (c) and women whose pre-pregnancy BMI was $18.5-24.9 \mathrm{~kg} / \mathrm{m}^{2}$. The study-specific WMD and $95 \% \mathrm{Cl}$ are represented by the grey square and horizontal line, respectively; the size of the data marker (grey square) is proportional to the weight of the study in the metaanalysis. The centre of the open diamond presents the pooled WMD and its width represents the pooled $95 \% \mathrm{Cl}$

association between GWG and PPWR are unknown, the present meta-analyses and the previous studies suggest that inadequate GWG affected PPWR, which decreased over postpartum time ${ }^{(22,28)}$. However, the potential beneficial effect of inadequate GWG on PPWR should be balanced against the potential risks of inadequate GWG for underweight women because underweight women would probably benefit from weight gain. Previous studies reported that nearly $35 \%$ of underweight women gained less weight than the recommended values. These women already had low BMI; therefore, inadequate weight gain increased the risk of complications, such as osteoporosis, preterm delivery and having a low-birth-weight infant ${ }^{(29)}$.

For women with excess GWG, stratification of postpartum time spans showed a U-shaped trend for this additional weight retention, being higher at the early postpartum, then declining by 1 year postpartum and then increasing again by 21 years postpartum. However, this result should be viewed with caution because it is based on the results of only two studies at 1-3 years postpartum $^{(13,15)}$ and two studies at more than 15 years postpartum $^{(9,11)}$. In addition, information on the time period between 3 and 15 years is unavailable. Mannan et al. also found a U-shaped trend for this additional weight retention, being higher at the early years postpartum, then declining by 8.5 years postpartum and then increasing again by 21 years postpartum ${ }^{(28)}$. The present research and the previous studies suggest that women who gained excess weight during pregnancy tended to have a greater PPWR in the long term. In the long term, these women are at greater risks of having an increased BMI and becoming overweight or obese postpartum.

Meta-analyses on GWG and PPWR in the present review were limited to studies that measured GWG in relation to IOM categories, which included pre-pregnancy BMI and can assess GWG independently of pre-pregnancy BMI. The effect of high and low GWG stratified by prepregnancy BMI on PPWR would be interesting to analyse. Unfortunately, only four of the included studies provided additional data on GWG stratified by pre-pregnancy BMI. Two studies suggested a marginally higher effect of high GWG on PPWR in mothers in a high-weight category ${ }^{(7,14)}$. The other two studies reported that when stratified by pre-pregnancy BMI and total weight gain adherence, only women with pre-pregnancy BMI in the normal or overweight ranges who gained a total weight that was greater than the recommended value retained more weight in the postpartum period than the women who met the total weight gain recommendations ${ }^{(8,19)}$. Begum et al. reported that $80 \%$ of the women with a BMI in the overweight or obese group gained a total weight that was greater than the recommended value, indicating that higher prepregnancy BMI is a significant predictor of excessive weight gain during pregnancy ${ }^{(19)}$. These results suggest that tools and intervention programmes have been developed to promote healthy weight gain for pregnant women; however, greater efforts should be exerted on women with high pre-pregnancy BMI.

The limitations of the present study should be considered. There exist a number of known determinants for PPWR, 
such as breast-feeding ${ }^{(12,13)}$, diet, physical activity and other lifestyle factors ${ }^{(30,31)}$. It is unclear whether these potential confounders might be more relevant because adjustment for confounding was attempted in only one of these studies. Therefore, we cannot exclude residual confounding of original studies in the meta-analysis. The present investigation was also limited by the low number of long-term studies, which weakened the reliability of long-term outcomes.

\section{Conclusion}

The meta-analysis results indicated that weight gain in pregnancy can lead to short- and long-term postpartum weight imbalance. These data support the recommendations of the US IOM regarding the importance of adequate weight gain avoiding short- and long-term high PPWR. The substantial association between excess GWG and PPWR indicates that high-quality confirmatory studies and studies on interventions based on diet and physical activity are clearly needed to address the issue of excess weight gain during pregnancy.

\section{Acknowledgements}

Financial support: This research project was sponsored by Nestlé Research Center. This research project was a collaborative work between Peking University and Nestlé. The views and opinions expressed in this manuscript are those of the authors and do not necessarily reflect the opinions and recommendations of Nestlé. Conflict of interest: None. Authorship: K.R., D.M., X.H., X.Q., J.W. and P.W. participated in the design of the manuscript. K.R., D.M., I.M.Y.S., K.Y. and Y.N. participated in performing the research and statistical analysis. All authors read and approved the final manuscript. Ethics of buman subject participation: Ethical approval was not required.

\section{References}

1. Flegal KM, Graubard BI, Williamson DF et al. (2007) Causespecific excess deaths associated with underweight, overweight, and obesity. JAMA 98, 2028-2037.

2. Zhang J, Huang Q, Yu M et al. (2013) Prevalence, awareness, medication, control, and risk factors associated with hypertension in Bai ethnic group in rural China: the Yunnan Minority Eye Study. PLoS One 8, e70886.

3. Harris HE \& Ellison GTH (1997) Do the changes in energy balance that occur during pregnancy predispose parous women to obesity? Nutr Res Rev 10, 57-81.

4. Williamson DF, Madans J, Pamuk E et al. (1994) A prospective study of childbearing and 10-year weight gain in US White women 25 to 45 years of age. Int J Obes Relat Metab Disord 18, 561-569.

5. Gunderson EP \& Abrams B (2000) Epidemiology of gestational weight gain and body weight changes after pregnancy. Epidemiol Rev 22, 261-274.
6. Lederman SA, Alfasi G \& Deckelbaum RJ (2002) Pregnancy associated obesity in black women in New York City. Matern Child Health J 6, 37-42.

7. Lowell H \& Miller DC (2010) Weight gain during pregnancy: adherence to Health Canada's guidelines. Health Rep $\mathbf{2 1}$, 31-36.

8. Rode L, Kjaergaard H, Ottesen B et al. (2012) Association between gestational weight gain according to body mass index and postpartum weight in a large cohort of Danish women. Matern Child Health J 16, 406-413.

9. Mamun AA, Kinarivala M, O'Callaghan MJ et al. (2010) Associations of excess weight gain during pregnancy with longterm maternal overweight and obesity: evidence from $21 \mathrm{y}$ postpartum follow-up. Am J Clin Nutr 91, 1336-1341.

10. Walker LO, Timmerman GM, Sterling BS et al. (2004) Do low-income women attain their pre-pregnant weight by the 6th week of postpartum? Ethn Dis 14, 119-126.

11. Amorim AR, Rossner S, Neovius M et al. (2007) Does excess pregnancy weight gain constitute a major risk for increasing long-term BMI? Obesity (Silver Spring) 15, 1278-1286.

12. Krause KM, Lovelady CA, Peterson BL et al. (2010) Effect of breast-feeding on weight retention at 3 and 6 months postpartum: data from the North Carolina WIC programme. Public Health Nutr 13, 2019-2026.

13. Østbye T, Krause KM, Swamy GK et al. (2010) Effect of breastfeeding on weight retention from one pregnancy to the next: results from the North Carolina WIC program. Prev Med 51, 368-372.

14. Kac G, Benicio MH, Velasquez-Melendez G et al. (2004) Gestational weight gain and prepregnancy weight influence postpartum weight retention in a cohort of Brazilian women. J Nutr 134, 661-666.

15. Maddah M \& Nikooyeh B (2009) Weight retention from early pregnancy to three years postpartum: a study in Iranian women. Midwifery 25, 731-737.

16. Gould Rothberg BE, Magriples U, Kershaw TS et al. (2011) Gestational weight gain and subsequent postpartum weight loss among young, low-income, ethnic minority women. Am J Obstet Gynecol 204, 52.e1-e11.

17. Baker JL, Gamborg M, Heitmann BL et al. (2008) Breastfeeding reduces postpartum weight retention. Am J Clin Nutr 88, 1543-1551.

18. Huang TT, Wang HS \& Dai FT (2010) Effect of prepregnancy body size on postpartum weight retention. Midwifery 26, 222-231.

19. Begum F, Colman I, McCargar LJ et al. (2012) Gestational weight gain and early postpartum weight retention in a prospective cohort of Alberta women. J Obstet Gynaecol Can 34, 637-647.

20. Stroup DF, Berlin JA, Morton SC et al. (2000) Meta-analysis of observational studies in epidemiology: a proposal for reporting. Meta analysis Of Observational Studies in Epidemiology (MOOSE) group. JAMA 283, 2008-2012.

21. Wells GA, Shea B, O'Connell D et al. (2011) The NewcastleOttawa Scale (NOS) for assessing the quality of nonrandomised studies in meta-analyses. http://www.ohri.ca/ programs/clinical_epidemiology/oxford.asp (accessed November 2012)

22. Kac G, Benicio MH, Velasquez-Melendez G et al. (2004) Nine months postpartum weight retention predictors for Brazilian women. Public Health Nutr 7, 621-628.

23. Scholl TO, Hediger ML, Schall JI et al. (1995) Gestational weight gain, pregnancy outcome, and postpartum weight retention. Obstet Gynecol 86, 423-427.

24. Koh H, Ee TX, Malhotra R et al. (2013) Predictors and adverse outcomes of inadequate or excessive gestational weight gain in an Asian population. J Obstet Gynaecol Res 39, $905-913$.

25. Althuizen E, van Poppel MN, de Vries JH et al. (2011) Postpartum behaviour as predictor of weight change from 
before pregnancy to one year postpartum. BMC Public Health 16, 165.

26. Oken E, Kleinman KP, Belfort MB et al. (2009) Associations of gestational weight gain with short- and longer-term maternal and child health outcomes. Am J Epidemiol 170, 173-180.

27. Nehring I, Schmoll S, Beyerlein A et al. (2011) Gestational weight gain and long-term postpartum weight retention: a meta-analysis. Am J Clin Nutr 94, 1225-1231.

28. Mannan M, Doi SA \& Mamun AA (2013) Association between weight gain during pregnancy and postpartum weight retention and obesity: a bias-adjusted meta-analysis. Nutr Rev 71, 343-352.

29. Wise LA, Palmer JR, Heffner LJ et al. (2010) Prepregnancy body size, gestational weight gain, and risk of preterm birth in African-American women. Epidemiology 21, 243-252.

30. Herring SJ, Rich-Edwards JW, Oken E et al. (2008) Association of postpartum depression with weight retention 1 year after childbirth. Obesity (Silver Spring) 16, 1296-1301.

31. Oken E, Taveras EM, Popoola FA et al. (2007) Television, walking, and diet: associations with postpartum weight retention. Am J Prev Med 32, 305-311. 